\title{
DESCRIPTOR FRACTIONAL LINEAR SYSTEMS WITH REGULAR PENCILS
}

\author{
TADEUSZ KACZOREK
}

Faculty of Electrical Engineering

Białystok Technical University, ul. Wiejska 45D, 15-351 Białystok, Poland

e-mail:kaczorek@isep.pw.edu.pl

\begin{abstract}
Methods for finding solutions of the state equations of descriptor fractional discrete-time and continuous-time linear systems with regular pencils are proposed. The derivation of the solution formulas is based on the application of the $\mathcal{Z}$ transform, the Laplace transform and the convolution theorems. Procedures for computation of the transition matrices are proposed. The efficiency of the proposed methods is demonstrated on simple numerical examples.
\end{abstract}

Keywords: descriptor system, fractional, system, regular pencil.

\section{Introduction}

Descriptor (singular) linear systems with regular pencils have been considered in many papers and books (Dodig and Stosic, 2009; Wang, 2012, Dai, 1989; Fahmy and O'Reill, 1989; Kaczorek, 2004; 1992; 2007a; 2007b; Kucera and Zagalak, 1988, Luenberger, 1978; Van Dooren, 1979). The eigenvalue and invariant assignment by state and output feedbacks was investigated by Dodig and Stosic (2009), Wang (2012), Dai (1989), Fahmy and O'Reill (1989) as well as Kaczorek (2004; 1992), while the realization problem for singular positive continuous-time systems with delays was discussed by Kaczorek (2007b). The computation of Kronecker's canonical form of a singular pencil was analyzed by Van Dooren (1979). A delay dependent criterion for a class of descriptor systems with delays varying in intervals was proposed by Wang (2012).

Fractional positive continuous-time linear systems were addressed by Kaczorek (2008), along with positive linear systems with different fractional order (Kaczorek, 2007a). A new concept of practical stability of positive fractional 2D systems was proposed by Kaczorek (2010b), who also presented an analysis of fractional linear electrical circuits (Kaczorek, 2012a) and some selected problems in the theory of fractional linear systems (Kaczorek, 2011b).

A new class of descriptor fractional linear systems and electrical circuits was introduced, their solution of state equations was derived and a method for decomposition of the descriptor fractional linear systems with regular pencils into dynamic and static parts was proposed by Kaczorek (2012a), who also considered positive fractional continuous-time linear systems with singular pencils (Kaczorek, 2012b). Fractional-order iterative learning control for fractional-order systems was addressed by Yan et al. (2011c).

In this paper, methods of finding solutions of the state equations of descriptor fractional discrete-time and continuous-time linear systems with regular pencils will be proposed.

The paper is organized as follows. In Section 2 the solution to the state equation of the descriptor system is derived using the method based on the $\mathcal{Z}$ transform and the convolution theorem. A method for computation of the transition matrix is proposed and illustrated on a simple numerical example in Section 3. In Section 4 the proposed method is extended to continuous-time linear systems. Concluding remarks are given in Section 5.

The following notation will be used: $\mathbb{R}$ is the set of real numbers, $\mathbb{R}^{n \times m}$ is the set of real $n \times m$ matrices and $\mathbb{R}^{n}=\mathbb{R}^{n \times 1}, \mathcal{Z}_{+}$is the set of nonnegative integers, $I_{n}$ is the $n \times n$ identity matrix.

\section{Discrete-time fractional linear systems}

Consider the descriptor fractional discrete-time linear system

$$
\begin{aligned}
E \Delta^{\alpha} x_{i+1} & =A x_{i}+B u_{i}, \\
& i \in \mathbb{Z}_{+}=\{0,1,2, \ldots\}, \quad 0<\alpha<1,
\end{aligned}
$$


where $\alpha$ is the fractional order, $x_{i} \in \mathbb{R}^{n}$ is the state vector $u_{i} \in \mathbb{R}^{m}$ is the input vector and $E, A \in \mathbb{R}^{n \times n}, B \in$ $\mathbb{R}^{n \times m}$. It is assumed that $\operatorname{det} E=0$, but the pencil ( $E$, $A)$ is regular, i.e.,

$$
\operatorname{det}[E z-A] \neq 0 \quad \text { for some } z \in \mathbb{C} .
$$

Without lost of generality we may assume that

$$
E=\left[\begin{array}{cc}
E_{1} & 0 \\
0 & 0
\end{array}\right] \in \mathbb{R}^{n \times n}, \quad E_{1} \in \mathbb{R}^{r \times r}
$$

and

$$
\operatorname{rank} E_{1}=\operatorname{rank} E=r<n .
$$

Admissible boundary conditions for (1) are given by $x_{0}$. The fractional difference of the order $\alpha \in[0,1)$ is defined by

$$
\Delta^{\alpha} x_{i}=\sum_{k=0}^{i} c_{k} x_{i-k}
$$

where

$$
c_{k}=(-1)^{k}\left(\begin{array}{c}
\alpha \\
k
\end{array}\right), \quad k=0,1, \ldots
$$

and

$$
\begin{aligned}
& \left(\begin{array}{l}
\alpha \\
k
\end{array}\right) \\
& =\left\{\begin{array}{cll}
1 & \text { for } & k=0, \\
\frac{\alpha(\alpha-1) \ldots(\alpha-k+1)}{k !} & \text { for } & k=1,2, \ldots
\end{array}\right.
\end{aligned}
$$

Substitution of (4a) into (1) yields

$$
E x_{i+1}=F x_{i}+\sum_{k=2}^{i+1} c_{k} x_{i-k+1}+B u_{i}, \quad i \in \mathbb{Z}_{+},
$$

where $F=A-E c_{1}=A-E \alpha$.

Applying to (5) the $\mathcal{Z}$-transform and taking into account that (Kaczorek, 1992)

$$
\begin{aligned}
& \mathcal{Z}\left[x_{i-p}\right] \\
& =z^{-p} X(z)+z^{-p} \sum_{j=-1}^{-p} x_{j} z^{-j}, \quad p=1,2, \ldots,
\end{aligned}
$$

we obtain

$$
X(z)=[E x-F]^{-1}\left\{E x_{0} z-H(z)+B U(z)\right\},
$$
where

$$
\begin{aligned}
& X(z)=\mathcal{Z}\left[x_{i}\right]=\sum_{i=0}^{\infty} x_{i} z^{-i} \\
& U(z)=\mathcal{Z}\left[u_{i}\right]=\sum_{i=0}^{\infty} u_{i} z^{-i} \\
& H(z)=\mathcal{Z}\left[h_{i}\right], \quad h_{i}=\sum_{k=2}^{i+1} E c_{k} x_{i-k+1} .
\end{aligned}
$$

Let

$$
[E x-F]^{-1}=\sum_{j=-\mu}^{\infty} \psi_{j} z^{-(j+1)}
$$

where $\mu$ is the positive integer defined by the pair $(E, A)$ (Kaczorek, 1992; Van Dooren, 1979). Comparison of the coefficients at the same powers of $z$ of the equality

$$
\begin{aligned}
& {[E x-F]\left(\sum_{j=-\mu}^{\infty} \psi_{j} z^{-(j+1)}\right)} \\
& =\left(\sum_{j=-\mu}^{\infty} \psi_{j} z^{-(j+1)}\right)[E x-F]=I_{n}
\end{aligned}
$$

yields

$$
E \psi_{-\mu}=\psi_{-\mu} E=0
$$

and

$$
\begin{aligned}
& E \psi_{k}+E \psi_{k+1} \\
& =\psi_{k} E+\psi_{k-1} E \\
& =\left\{\begin{array}{ccc}
I_{n} & \text { for } \quad k=0, \\
0 & \text { for } \quad k=1-\mu, 2-\mu, \ldots,-1,1,2, \ldots
\end{array}\right.
\end{aligned}
$$

From (9b) and (9c) we have the matrix equation

$$
G\left[\begin{array}{c}
\psi_{0 \mu} \\
\psi_{1 N}
\end{array}\right]=\left[\begin{array}{c}
V \\
0
\end{array}\right]
$$

where

$$
\begin{aligned}
& G=\left[\begin{array}{cc}
G_{1} & 0 \\
G_{21} & G_{2}
\end{array}\right] \in \mathbb{R}^{(N+\mu+1) n \times(N+\mu+1) n}, \\
& G_{21}=\left[\begin{array}{cccc}
0 & \ldots & 0 & F \\
0 & \ldots & 0 & 0 \\
\vdots & \ldots & \vdots & \vdots \\
0 & \ldots & 0 & 0
\end{array}\right] \in \mathbb{R}^{N n \times(\mu+1) n} \\
& G_{1}=\left[\begin{array}{ccccccc}
E & 0 & 0 & \ldots & 0 & 0 & 0 \\
F & E & 0 & \ldots & 0 & 0 & 0 \\
0 & F & E & \ldots & 0 & 0 & 0 \\
\vdots & \vdots & \vdots & \ldots & \vdots & \vdots & \vdots \\
0 & 0 & 0 & \ldots & F & E & 0 \\
0 & 0 & 0 & \ldots & 0 & F & E
\end{array}\right] \\
& \in \mathbb{R}^{(\mu+1) n \times(\mu+1) n}, \\
& G_{2}=\left[\begin{array}{ccccccc}
E & 0 & 0 & \ldots & 0 & 0 & 0 \\
F & E & 0 & \ldots & 0 & 0 & 0 \\
0 & F & E & \ldots & 0 & 0 & 0 \\
\vdots & \vdots & \vdots & \ldots & \vdots & \vdots & \vdots \\
0 & 0 & 0 & \ldots & F & E & 0 \\
0 & 0 & 0 & \ldots & 0 & F & E
\end{array}\right] \in \mathbb{R}^{N n \times N n}
\end{aligned}
$$




$$
\begin{aligned}
\psi_{0 \mu}= & {\left[\begin{array}{c}
\psi_{-\mu} \\
\psi_{1-\mu} \\
\vdots \\
\psi_{0}
\end{array}\right] \in \mathbb{R}^{(\mu+1) n \times n}, } \\
\psi_{1 N}= & {\left[\begin{array}{c}
\psi_{1} \\
\psi_{2} \\
\vdots \\
\psi_{N}
\end{array}\right] \in \mathbb{R}^{N n \times n}, } \\
V= & {\left[\begin{array}{c}
0 \\
\vdots \\
0 \\
I_{n}
\end{array}\right] \in \mathbb{R}^{(\mu+1) n \times n} . }
\end{aligned}
$$

Equation (10a) has the solution

$$
\left[\begin{array}{c}
\psi_{0 \mu} \\
\psi_{1 N}
\end{array}\right]
$$

for given $G$ and $V$ if and only if

$$
\operatorname{rank}\left\{G,\left[\begin{array}{c}
V \\
0
\end{array}\right]\right\}=\operatorname{rank} G .
$$

It is easy to show that the condition (11) is satisfied if the condition (2) is met.

Substituting (8) into (7a) we obtain

$$
X(z)=\left(\sum_{j=-\mu}^{\infty} \psi_{j} z^{-(j+1)}\right)\left[E x_{0} z-H(z)+B U(z)\right] .
$$

Applying the inverse transform $\mathcal{Z}^{-1}$ and the convolution theorem to [12, we obtain

$$
\begin{aligned}
x_{i}= & \psi_{i} E x_{0}-\sum_{k=0}^{i+\mu-1} \psi_{i-k-1} \sum_{j=2}^{k+1} c_{j} x_{k-j+1} \\
& +\sum_{k=0}^{i+\mu-1} \psi_{i-k-1} B u_{k} .
\end{aligned}
$$

To find the solution to Eqn. (1), first we compute the transition matrices $\psi_{j}$ for $j=-\mu, 1-\mu, \ldots, 1,2, \ldots$ and next, using (13), we obtain the desired solution.

\section{Computation of transition matrices}

To compute the transition matrices $\psi_{k}$ for $k=-\mu, 1-$ $\mu, \ldots, N, \ldots$, the following procedure is recommended.

\section{Procedure 1.}

Step 1. Find a solution $\psi_{0 \mu}$ of the equation

$$
G_{1} \psi_{0 \mu}=V,
$$

where $G_{1}, \psi_{0 \mu}$ and $V$ are defined by (10b). Note that, if the matrix $E$ has the form (3), then the first $r$ rows of the matrix $\psi_{0 \mu}$ are zero and its $n-r$ last rows are arbitrary.
Step 2. Choose $n-r$ arbitrary rows of the matrix $\psi_{0}$ so that

$$
\begin{array}{r}
\operatorname{rank}\left\{\left[\begin{array}{cc}
E & 0 \\
F & E
\end{array}\right],\left[\begin{array}{c}
I_{n}-F \psi_{-1} \\
0
\end{array}\right]\right\} \\
=\operatorname{rank}\left[\begin{array}{cc}
E & 0 \\
F & E
\end{array}\right]
\end{array}
$$

and the equation

$$
\left[\begin{array}{ll}
E & 0 \\
F & E
\end{array}\right]\left[\begin{array}{l}
\psi_{0} \\
\psi_{1}
\end{array}\right]=\left[\begin{array}{c}
I_{n}-F \psi_{-1} \\
0
\end{array}\right]
$$

has a solution with arbitrary last $n-r$ rows of the matrix $\psi_{1}$.

Step 3. Knowing $\psi_{0 \mu}$, choose the last $n-r$ rows of the matrix $\psi_{1}$ so that

$$
\operatorname{rank}\left\{\left[\begin{array}{cc}
E & 0 \\
F & E
\end{array}\right],\left[\begin{array}{c}
F \psi_{0} \\
0
\end{array}\right]\right\}=\operatorname{rank}\left[\begin{array}{cc}
E & 0 \\
F & E
\end{array}\right]
$$

and the equation

$$
\left[\begin{array}{ll}
E & 0 \\
F & E
\end{array}\right]\left[\begin{array}{l}
\psi_{1} \\
\psi_{2}
\end{array}\right]=-\left[\begin{array}{l}
F \\
0
\end{array}\right] \psi_{0}
$$

has a solution with arbitrary last $n-r$ rows of the matrix $\psi_{2}$. Repeating the last step for

$$
\left[\begin{array}{l}
\psi_{2} \\
\psi_{3}
\end{array}\right],\left[\begin{array}{ll}
\psi_{3} & \\
\psi_{4}
\end{array}, \ldots\right]
$$

we may compute the desired matrices $\psi_{k}$ for $k=-\mu, 1-$ $\mu, \ldots$.

The details of the procedure will be shown on the following example.

Example 1. Find the solution to Eqn. (1) for $\alpha=0.5$ with the matrices

$$
E=\left[\begin{array}{ll}
1 & 0 \\
0 & 0
\end{array}\right], \quad A=\left[\begin{array}{cc}
0 & 0 \\
1 & -2
\end{array}\right], \quad B=\left[\begin{array}{l}
1 \\
2
\end{array}\right]
$$

and the initial condition

$$
x_{0}=\left[\begin{array}{l}
1 \\
0
\end{array}\right]
$$

and $u_{i}, \quad i \in \mathbb{Z}_{+}$.

In this case the pencil (2) of (18) is regular since

$$
\operatorname{det}[E z-A]=\left|\begin{array}{cc}
z & 0 \\
-1 & 2
\end{array}\right|=2 z
$$

$\mu=1$ and

$$
F=[E \alpha-A]=\left[\begin{array}{cc}
\alpha & 0 \\
-1 & 2
\end{array}\right]=\left[\begin{array}{cc}
0.5 & 0 \\
-1 & 2
\end{array}\right] .
$$


Using Procedure 1, we obtain the following.

Step 1. In this case Eqn. (14) has the form

$$
\left[\begin{array}{cccc}
1 & 0 & 0 & 0 \\
0 & 0 & 0 & 0 \\
\alpha & 0 & 1 & 0 \\
-1 & 2 & 0 & 0
\end{array}\right]\left[\begin{array}{c}
\psi_{-1} \\
\psi_{0}
\end{array}\right]=\left[\begin{array}{ll}
0 & 0 \\
0 & 0 \\
1 & 0 \\
0 & 1
\end{array}\right]
$$

and its solution with the arbitrary second row $\left[\begin{array}{ll}\psi_{21}^{0} & \psi_{22}^{0}\end{array}\right]$ of $\psi_{0}$ is given by

$$
\left[\begin{array}{c}
\psi_{-1} \\
\psi_{0}
\end{array}\right]=\left[\begin{array}{cc}
0 & 0 \\
0 & 0.5 \\
1 & 0 \\
\psi_{21}^{0} & \psi_{22}^{0}
\end{array}\right]
$$

Step 2. We choose the row [ $\left.\psi_{21}^{0} \quad \psi_{22}^{0}\right]$ of $\psi_{0}$ so that (15) holds, i.e.,

$$
\begin{aligned}
& \operatorname{rank}\left[\begin{array}{cccccc}
1 & 0 & 0 & 0 & 1 & 0 \\
0 & 0 & 0 & 0 & 0 & 0 \\
\alpha & 0 & 1 & 0 & 0 & 0 \\
-1 & 2 & 0 & 0 & 0 & 0
\end{array}\right] \\
&=\operatorname{rank}\left[\begin{array}{cccc}
1 & 0 & 0 & 0 \\
0 & 0 & 0 & 0 \\
\alpha & 0 & 1 & 0 \\
-1 & 2 & 0 & 0
\end{array}\right],
\end{aligned}
$$

and the equation

$$
\left[\begin{array}{cccc}
1 & 0 & 0 & 0 \\
0 & 0 & 0 & 0 \\
\alpha & 0 & 1 & 0 \\
-1 & 2 & 0 & 0
\end{array}\right]\left[\begin{array}{l}
\psi_{0} \\
\psi_{1}
\end{array}\right]=\left[\begin{array}{ll}
0 & 0 \\
0 & 0 \\
1 & 0 \\
0 & 1
\end{array}\right]
$$

has the solution

$$
\left[\begin{array}{l}
\psi_{0} \\
\psi_{1}
\end{array}\right]=\left[\begin{array}{cc}
1 & 0 \\
0.5 & 0 \\
-\alpha & 0 \\
\psi_{21}^{1} & \psi_{22}^{1}
\end{array}\right]
$$

with the second arbitrary row [ $\psi_{21}^{1} \quad \psi_{22}^{1}$ ] of $\psi_{1}$. Step 3. We choose [ $\left.\psi_{21}^{1} \quad \psi_{22}^{1}\right]$ so that the equation

$$
\left[\begin{array}{cccc}
1 & 0 & 0 & 0 \\
0 & 0 & 0 & 0 \\
\alpha & 0 & 1 & 0 \\
-1 & 2 & 0 & 0
\end{array}\right]\left[\begin{array}{cc}
-\alpha & 0 \\
\psi_{21}^{1} & \psi_{22}^{1} \\
\alpha^{2} & 0 \\
\psi_{21}^{2} & \psi_{22}^{2}
\end{array}\right]=-\left[\begin{array}{ll}
\alpha & 0 \\
0 & 0 \\
0 & 0 \\
0 & 0
\end{array}\right]
$$

has the solution

$$
\left[\begin{array}{l}
\psi_{1} \\
\psi_{2}
\end{array}\right]=\left[\begin{array}{cc}
-\alpha & 0 \\
-0.5 \alpha & 0 \\
\alpha^{2} & 0 \\
\psi_{21}^{2} & \psi_{22}^{2}
\end{array}\right]
$$

with arbitrary [ $\left.\psi_{21}^{2} \quad \psi_{22}^{2}\right]$

Continuing the procedure, we obtain

$$
\psi_{-1}=\left[\begin{array}{cc}
0 & 0 \\
0 & 0.5
\end{array}\right], \quad \psi_{k}=(-1)^{k}\left[\begin{array}{cc}
\alpha^{k} & 0 \\
0.5 \alpha^{k} & 0
\end{array}\right]
$$

for $k=0,1, \ldots$

Using (13), (18) and (20), we obtain the desired solution of the form

$$
\begin{aligned}
x_{i}= & \psi_{i}\left[\begin{array}{l}
1 \\
0
\end{array}\right]-\sum_{k=0}^{i} \psi_{i-k-1} \sum_{j=2}^{k+1}\left[\begin{array}{ll}
1 & 0 \\
0 & 0
\end{array}\right] c_{j} x_{k-j+1} \\
& +\sum_{k=0}^{i} \psi_{i-k-1}\left[\begin{array}{l}
1 \\
2
\end{array}\right] u_{k}
\end{aligned}
$$

where $c_{j}$ are defined by (4b).

\section{Continuous-time fractional linear systems}

Consider the descriptor fractional continuous-time linear system described by the state equations

$$
\begin{aligned}
E D^{\alpha} x(t)= & A x(t)+B u(t), \\
& n-1<\alpha \leq n \in\{1,2, \ldots\} \\
y(t)= & C x(t)+D u(t),
\end{aligned}
$$

where $D^{\alpha}$ is the Caputo differentiation operator, $x(t) \in$ $\mathbb{R}^{n}, u(t) \in \mathbb{R}^{m}, y(t) \in \mathbb{R}^{p}$ are the state, input and output vectors and $E, A \in \mathbb{R}^{n \times n}, B \in \mathbb{R}^{n \times m}, C \in \mathbb{R}^{p \times n}, D \in$ $\mathbb{R}^{p \times m}$.

It is assumed that $\operatorname{det} E=0$ and the pencil $(E, A)$ is regular, i.e.,

$$
\operatorname{det}[E \lambda-A] \neq 0 \quad \text { for some } \quad \lambda \in \mathcal{C} .
$$

Admissible initial conditions for (30a) are given by

$$
x^{(k)}(0)=x_{k, 0} \quad \text { for } \quad k=0,1, \ldots, n-1 .
$$

Applying the Laplace transform $(\mathcal{L})$ to Eqn. (30a), we obtain (Kaczorek, 2011b)

$$
\left[E s^{\alpha}-A\right] X(s)=B U(s)+\sum_{k=0}^{n-1} s^{\alpha-k-1} x_{k, 0},
$$

where $X(s)=\mathcal{L}[x(t)]$ and $U(s)=\mathcal{L}[u(t)]$. If the condition (31) is satisfied, then from (33) we obtain

$$
X(s)=\left[E s^{\alpha}-A\right]^{-1}\left(B U(s)+\sum_{k=0}^{n-1} s^{\alpha-k-1} x_{k, 0}\right) .
$$


In a particular case when $\operatorname{det} E \neq 0$, from (34) we have

$$
\begin{aligned}
X(s)= & \sum_{i=0}^{\infty}\left(E^{-1} A\right)^{i} E^{-1} s^{-(i+1) \alpha} \\
& \times\left(B U(s)+\sum_{k=0}^{n-1} s^{\alpha-k-1} x_{k, 0}\right)
\end{aligned}
$$

since

$$
\begin{aligned}
{\left[E s^{\alpha}-A\right]^{-1} } & =\left[E s^{\alpha}\left(I_{n}-\left(E s^{\alpha}\right)^{-1}\right) A\right]^{-1} \\
& =\sum_{i=0}^{\infty}\left(E^{-1} A\right)^{i} E^{-1} s^{-(i+1) \alpha} .
\end{aligned}
$$

Using the inverse Laplace transform $\left(\mathcal{L}^{-1}\right)$ and the convolution theorem, we obtain

$$
\begin{aligned}
x(t)= & \mathcal{L}^{-1}[X(s)] \\
= & \sum_{i=0}^{\infty}\left[\int_{0}^{t}\left(E^{-1} A\right)^{i} \frac{(t-\tau)^{(i+1) \alpha-1}}{\Gamma[(i+1) \alpha]} E^{-1} B u(\tau) \mathrm{d} \tau\right. \\
& \left.+\sum_{k=0}^{n-1} \frac{t^{i \alpha+k}}{\Gamma(i \alpha+k+1)}\left(E^{-1} A\right)^{i} x_{k, 0}\right],
\end{aligned}
$$

where $\Gamma(\alpha)$ is the gamma function (Kaczorek, 2011b).

Therefore, the following theorem for $\operatorname{det} E \neq 0$ has been proved.

Theorem 1. The solution of Eqn. (30a) for $\operatorname{det} E \neq 0$ and the initial conditions (32) is given by (36).

If $E=I_{n}$, then (36) takes the form (Kaczorek, $2011 b)$

$$
x(t)=\sum_{l=1}^{n} \Phi_{l}(t) x^{(l-1)}\left(0^{+}\right)+\int_{0}^{t} \Phi(t-\tau) B u(\tau) \mathrm{d} \tau,
$$

where

$$
\begin{aligned}
\Phi_{l}(t) & =\sum_{k=0}^{\infty} \frac{A^{k} t^{(k \alpha+l)-1}}{\Gamma(k \alpha+l)}, \\
\Phi(t) & =\sum_{k=0}^{\infty} \frac{A^{k} t^{(k+1) \alpha-1}}{\Gamma[(k+1) \alpha]} .
\end{aligned}
$$

If $\operatorname{det} E=0$ but the pencil is regular (the condition (31) is met), then

$$
\left[E s^{\alpha}-A\right]^{-1}=\sum_{i=-\mu}^{\infty} T_{i} s^{-(i+1) \alpha}
$$

where $T_{i}$ satisfy the equality

$$
\begin{aligned}
& E T_{i}-A T_{i-1}=T_{i} E-T_{i-1} A \\
& = \begin{cases}I_{n} & \text { for } \quad i=0, \\
0 & \text { for } i \neq 0,\end{cases}
\end{aligned}
$$

and $T_{i}=0$ for $i<-\mu, T_{-\mu} E=E T_{-\mu}=0$.

The equality (40) follows from the comparison of the coefficients at the same powers of $s$ in the equality

$$
\begin{aligned}
& {\left[E s^{\alpha}-A\right]\left(\sum_{i=-\mu}^{\infty} T_{i} s^{-(i+1) \alpha}\right)} \\
& \quad=\left(\sum_{i=-\mu}^{\infty} T_{i} s^{-(i+1) \alpha}\right)\left[E s^{\alpha}-A\right]=I_{n} .
\end{aligned}
$$

Substitution of (39) into (34) yields

$$
\begin{aligned}
X(s)= & \sum_{i=-\mu}^{\infty} T_{i}\left[s^{-(i+1) \alpha} B U(s)+\sum_{k=0}^{n-1} s^{-i \alpha-k-1} x_{k, 0}\right] \\
= & \sum_{i=0}^{\infty} T_{i}\left[s^{-(i+1) \alpha} B U(s)+\sum_{k=0}^{n-1} s^{-i \alpha-k-1} x_{k, 0}\right] \\
& +\sum_{i=1}^{\mu} T_{-i}\left[s^{(i+1) \alpha} B U(s)+\sum_{k=0}^{n-1} s^{i \alpha-k-1} x_{k, 0}\right] .
\end{aligned}
$$

Applying the inverse Laplace transform and the convolution theorem to 41, we obtain

$$
\begin{aligned}
x(t)= & \sum_{i=0}^{\infty}\left[\int_{0}^{t} T_{i} B \frac{(t-\tau)^{(i+1) \alpha-1}}{\Gamma[(i+1) \alpha]} B u(\tau) \mathrm{d} \tau\right. \\
& \left.+\sum_{k=0}^{n-1} T_{i} \frac{t^{i \alpha+k}}{\Gamma(i \alpha+k+1)} E x_{k, 0}\right] \\
& +\sum_{i=1}^{\mu} T_{-i}\left[B u(t)^{(i-1) \alpha}+\sum_{k=0}^{n-1} \delta^{(i \alpha-1)} E x_{k, 0}\right]
\end{aligned}
$$

or

$$
\begin{aligned}
x(t)= & \sum_{i=0}^{\infty}\left[\int_{0}^{t}\left(T_{0} A\right)^{i} T_{0} B \frac{(t-\tau)^{(i+1) \alpha-1}}{\Gamma[(i+1) \alpha]} u(\tau) \mathrm{d} \tau\right. \\
& \left.+\sum_{k=0}^{n-1}\left(T_{0} A\right)^{i} T_{0} \frac{t^{i \alpha+k}}{\Gamma(i \alpha+k+1)} x_{k, 0}\right] \\
& +\sum_{i=1}^{\mu} T_{-i}\left[B u(t)^{(i-1) \alpha}+\sum_{k=0}^{n-1} \delta^{(i \alpha-1)} E x_{k, 0}\right],
\end{aligned}
$$

since by 40) $T_{i}=\left(T_{0} A\right)^{i} T_{0}$ for $i=0,1, \ldots$ and $\delta^{(k)}$ is the $k$-th derivative of the delta impulse function $\delta$.

Therefore, the following theorem has been proved.

Theorem 2. If the condition (31) is satisfied, then the solution of Eqn. (30a) with the admissible initial conditions (32) is given by (42) or (43). 
In a particular case of $0<\alpha \leq 1$, from (43) we have

$$
\begin{aligned}
x(t)= & \sum_{i=0}^{\infty}\left(T_{0} A\right)^{i} T_{0}\left[\int_{0}^{t} B \frac{(t-\tau)^{(i+1) \alpha-1}}{\Gamma[(i+1) \alpha]} u(\tau) \mathrm{d} \tau\right. \\
& \left.+\frac{t^{i \alpha}}{\Gamma(i \alpha+1)} E x_{0}\right] \\
& +\sum_{i=1}^{\mu} T_{-i}\left[B u(t)^{(i-1) \alpha}+\delta^{(i \alpha-1)} E x_{0}\right] .
\end{aligned}
$$

To compute the matrices $T_{i}$ for $i=-\mu, 1-\mu, \ldots$, the procedure given in Section 3 is recommended.

Example 2. Consider Eqn. (30a) for $\alpha=0.5$ with the matrices

$$
E=\left[\begin{array}{ll}
1 & 0 \\
0 & 0
\end{array}\right], \quad A=\left[\begin{array}{cc}
0 & 0 \\
1 & -2
\end{array}\right], \quad B=\left[\begin{array}{l}
1 \\
2
\end{array}\right]_{(45)}
$$

and the zero initial condition $x_{0}=0$. The pencil is regular since

$$
\operatorname{det}[E \lambda-A]=\left|\begin{array}{cc}
\lambda & 0 \\
-1 & 2
\end{array}\right|=2 \lambda, \quad\left(\lambda=s^{\alpha}\right)
$$

and

$$
[E \lambda-A]^{-1}=\left[\begin{array}{cc}
\lambda^{-1} & 0 \\
0.5 \lambda^{-1} & 0.5
\end{array}\right]=T_{-1}+T_{0} \lambda^{-1}
$$

where

$$
T_{-1}=\left[\begin{array}{cc}
0 & 0 \\
0 & 0.5
\end{array}\right], \quad T_{0}=\left[\begin{array}{cc}
1 & 0 \\
0.5 & 0
\end{array}\right] .
$$

Using (42), (43) and (47b) we obtain

$$
\begin{aligned}
x(t) & =T_{0} B \int_{0}^{t} \frac{(t-\tau)^{-0.5}}{\Gamma(0.5)} u(\tau) \mathrm{d} \tau+T_{-1} B u(t) \\
& =\left[\begin{array}{c}
1 \\
0.5
\end{array}\right] \int_{0}^{t} \frac{(t-\tau)^{-0.5}}{\Gamma(0.5)} u(\tau) \mathrm{d} \tau+\left[\begin{array}{l}
0 \\
1
\end{array}\right] u(t) .
\end{aligned}
$$

\section{Concluding remarks}

New methods of finding solutions of the state equations of descriptor fractional discrete-time and continuous-time linear systems with regular pencils have been proposed. Derivation of the solution formulas has been based on the application of the $\mathcal{Z}$-transform, the Laplace transform and the convolution theorems. A procedure for computation of the transition matrices has been proposed and its application has been demonstrated on simple numerical examples. An open problem is the extension of the method for 2D descriptor fractional discrete and continuous-discrete linear systems.

\section{Acknowledgment}

This work was supported by the National Science Centre in Poland under the grant no. N N514 638940.

\section{References}

Dodig, M. and Stosic, M. (2009). Singular systems state feedbacks problems, Linear Algebra and Its Applications 431(8): $1267-1292$.

Dai, L. (1989). Singular Control Systems, Lectures Notes in Control and Information Sciences, Vol. 118, Springer-Verlag, Berlin.

Fahmy, M.H and O'Reill, J. (1989). Matrix pencil of closed-loop descriptor systems: Infinite-eigenvalues assignment, International Journal of Control 49(4): 1421-1431.

Gantmacher, F.R. (1960). The Theory of Matrices, Chelsea Publishing Co., New York, NY.

Kaczorek, T. (2012a). Descriptor fractional linear systems with regular pencils, Asian Journal of Control 15(4): 1-14.

Kaczorek, T. (2012b). Positive fractional continuous-time linear systems with singular pencils, Bulletin of the Polish Academy of Sciences: Technical Sciences 60(1): 9-12.

Kaczorek, T. (2011a). Positive linear systems consisting of $n$ subsystems with different fractional orders, IEEE Transactions on Circuits and Systems 58(7): 1203-1210.

Kaczorek, T. (2011b). Selected Problems of Fractional System Theory, Springer-Verlag, Berlin.

Kaczorek, T. (2010a). Positive linear systems with different fractional orders, Bulletin of the Polish Academy of Sciences: Technical Sciences 58(3): 453-458.

Kaczorek, T. (2010b). Practical stability and asymptotic stability of positive fractional 2D linear systems, Asian Journal of Control 12(2): 200-207.

Kaczorek, T. (2008). Fractional positive continuous-time linear systems and their reachability, International Journal of Applied Mathematics and Computer Science 18(2): 223-228, DOI: 10.2478/v10006-008-0020-0.

Kaczorek, T. (2007a). Polynomial and Rational Matrices. Applications in Dynamical Systems Theory, Springer-Verlag, London.

Kaczorek, T. (2007b). Realization problem for singular positive continuous-time systems with delays, Control and Cybernetics 36(1): 47-57.

Kaczorek, T. (2004). Infinite eigenvalue assignment by an output feedbacks for singular systems, International Journal of Applied Mathematics and Computer Science 14(1): 19-23. 
Kaczorek, T. (1992). Linear Control Systems, Vol. 1, Research Studies Press J. Wiley, New York, NY.

Kucera, V. and Zagalak, P. (1988). Fundamental theorem of state feedback for singular systems, Automatica 24(5): 653-658.

Luenberger, D.G. (1978). Time-invariant descriptor systems, $A u$ tomatica 14: 473-480.

Podlubny, I. (1999). Fractional Differential Equations, Academic Press, New York, NY.

Wang, C. (2012). New delay-dependent stability criteria for descriptor systems with interval time delay, Asian Journal of Control 14(1): 197-206.

Van Dooren, P. (1979). The computation of Kronecker's canonical form of a singular pencil, Linear Algebra and Its Applications 27: 103-140.

Yan L., YangQuan C., Hyo-Sung A., (2011c). Fractional-order iterative learning control for fractional-order systems, Asian Journal of Control 13(1): 54-63.

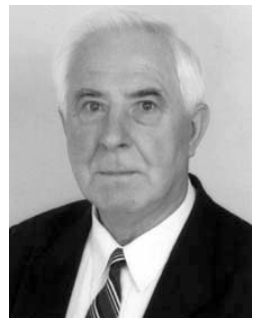

Tadeusz Kaczorek received the M.Sc., Ph.D. and D.Sc. degrees in electrical engineering from the Warsaw University of Technology in 1956, 1962 and 1964, respectively. In the years 1968-69 he was the dean of the Electrical Engineering Faculty, and in the period of 1970-73 he was a deputy rector of the Warsaw University of Technology. In 1971 he became a professor and in 1974 a full professor at the same university. Since 2003 he has been a professor at Białystok Technical University. In 1986 he was elected a corresponding member and in 1996 a full member of the Polish Academy of Sciences. In the years 1988-1991 he was the director of the Research Centre of the Polish Academy of Sciences in Rome. In 2004 he was elected an honorary member of the Hungarian Academy of Sciences. He has been granted honorary doctorates by nine universities. His research interests cover systems theory, especially singular multidimensional systems, positive multidimensional systems, singular positive $1 \mathrm{D}$ and $2 \mathrm{D}$ systems, as well as positive fractional $1 \mathrm{D}$ and $2 \mathrm{D}$ systems. He initiated research in the field of singular 2D, positive 2D and positive fractional linear systems. He has published 24 books (six in English) and over 1000 scientific papers. He has also supervised $69 \mathrm{Ph} . \mathrm{D}$. theses. He is the editor-in-chief of the Bulletin of the Polish Academy of Sciences: Technical Sciences and a member of editorial boards of ten international journals.

Received: 28 May 2012

Revised: 12 September 2012 